\title{
Investigation of the defects effect on energy absorption by the system under cyclic loadings
}

\author{
Igor Golovnev ${ }^{1}$, Elena Golovneva ${ }^{1, *}$, and Vasily Fomin ${ }^{1}$ \\ ${ }^{1}$ Khristianovich Institute of Theoretical and Applied Mechanics SB RAS, Novosibirsk 630090, Russia
}

\begin{abstract}
The paper presents the comparison of the energy absorption by perfect and amorphized nanorods under the cyclic loading. The calculations have been carried out with the molecular dynamic modeling. The cyclic loading is modeled by a periodical shift of a movable clamp along the $\mathrm{X}$ axis. The calculations are performed for the maximal clamp shift in respect to the equilibrium state, which corresponds to the relative deformation (equal to $0.3 \%$ ). Frequencies varied from $0.085 \mathrm{THz}$ to 10 THz. It has been discovered that both the dramatic growth of the maximal dispersion of atomic planes, and the dramatic growth of amplification of the maximal dispersion of atomic planes can be used as an index of the irreversible transformation of the crystal structure in the physical system differing from the perfect nanosized crystal. General behavior of the studied systems more depends on cyclic loading parameters than on the object crystal structure. Under the cyclic loading modeled with the aid of the periodical shift of the movable clamp, the frequency makes the critical impact on the results.
\end{abstract}

\section{Task statement}

The present work is addressed to the analysis of the energy absorption of the external action by a nanorod under the cyclic action and to the investigation of the effect of defects in the sample on this process. To solve the stated task, the numerical analysis of the processes in the nanosized nanorod has been performed at the cyclic uniaxial pull, by the molecular dynamics method.

In their previous work, the authors found the indices of the beginning defects in the nanorod; they are generated by the effect of the external harmonic uniaxial push-pull which is described by the periodical shift of the rod free end [1]. These results are used in the present work.

\footnotetext{
* Corresponding author: elena@itam.nsc.ru
} 


\section{Description of physical systems, clamp modeling and calculation techniques}

The perfect copper crystal with the face-centered cubic structure shaped as a rectangular parallelepiped, the crystal cell amount $\mathrm{n}_{\mathrm{x}}=50, \mathrm{n}_{\mathrm{y}}=\mathrm{n}_{\mathrm{z}}=5$ along respective axes is used as the basic perfect sample. The crystal is oriented toward the $X$ axis [100].

The sample with crystal lattice defects has been obtained as follows: the perfect nanorod with the sizes as given above, undergoes the impulse impact with its left edge, the impact direction is along the $\mathrm{X}$ axis, the clamps are not fixed. As a result, the crystal lattice has changed. The authors afforded to use the name "the amorphized crystal" for such a system.

For more detailed information about the processes in the crystal under the impulse impact and forming translational defects during this process, see [2,3].

To model the interatomic interaction, the potential based on the embedded atom method [4] is used.

Initial data were prepared as follows. Using the perfect crystal with the face-centered cubic structure and amorphized crystal as a base, the artificial viscosity method [5] was applied to determine the atom coordinates and impulses which correlate to the minimal potential energy of the structure. Hence obtained values of coordinates and impulses were used as the initial data for every following calculation.

The paper uses the commonly known Verlet velocity modification [6] of the second order of accuracy, with the time pitch $\tau=10^{-16} \mathrm{~s}$. It should be stressed that in the case of the isolated system, the energy error does not exceed $10^{-5} \%$ within the time interval of $50 \mathrm{ps}$.

Modeling of the leftmost immovable clamp and movable one assigning the cyclic shift has been described in detail by the authors in [1].

Basing on the authors' paper [7], devoted to the analysis of the cyclic loading amplitudes and frequencies effect on the energy absorption of the system disturbance, the following sets of parameters have been chosen for the comparison of the energy absorption in the nanorods of two different sizes (see Table 1).

Frequency $v=1 /\left(\mathrm{N}_{\omega} \cdot \tau\right)$, where $\tau$ is the calculated time pitch, $\mathrm{N}_{\omega}$ is the amount of time pitches in one oscillation period.

In the work, the energy analysis is performed for such characteristics as the variation of the total potential energy $\Delta \mathrm{U}_{\mathrm{t}}$ and variation of the chaotic component of the kinetic energy of atom motion $\Delta \mathrm{E}_{\mathrm{kin}}$, the nanorod temperature is $\mathrm{T}$. They show to the fullest extent possible, how the absorbed energy distributes under the influence of the external disturbance.

The crystal structure was studied with the aid of the common neighbor analysis in the visualization program OVITO (htto://ovito.org/) [8, 9].

Let us now consider the results for three sets of data which were obtained earlier in the authors' paper [7] (the cyclic loading amplitudes and frequencies see in Table 1); on the first stage, both compared systems are at the zero temperature.

Table 1. The cyclic loading amplitudes and frequencies.

\begin{tabular}{|c|c|}
\hline Frequency, $v$ & $0.5 \AA$ \\
\hline $0.085 \mathrm{THz}$ & No fracture up to $500 \mathrm{ps}$ \\
\hline $1 \mathrm{THz}$ & Fracture is visible at $10 \mathrm{ps}$. \\
\hline $10 \mathrm{THz}$ & Fracture occurs at $1 \mathrm{ps}$. \\
\hline
\end{tabular}




\section{Description of physical systems, clamp modeling and calculation techniques}

Let us compare both systems at the initial time instant, i.e. before any external loading is applied (Fig. 1).
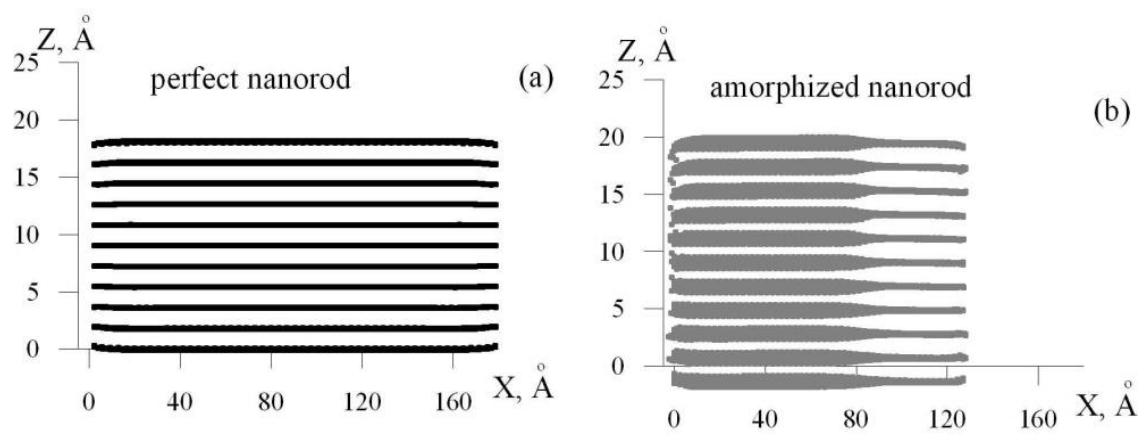

Fig. 1. View of the perfect (a) and amorphized (b) nanosized rod in the XZ plane.

The initial length of the perfect nanorod is $180 \AA$ (18 nm), and the initial length of the amorphized nanorod is about $130 \AA(13 \mathrm{~nm})$, which is the result of the impact directed along the $\mathrm{X}$ axis.

Figure 2 shows the same systems in the $\mathrm{YZ}$ plane, the view from the movable clamp side. It is evident from the figures that the impact resulted in the sample compression along the $\mathrm{X}$ axis, the atomic planes shifted.

The outlook of the nanorod end face (in the YZ plane) shows that for the amorphized crystal case, the accurate calculation of the area of the left immovable and right movable clamps is extremely difficult. At this stage of investigations, the area of the amorphized rod is calculated in the same way as for the perfect crystal: we find the coordinates of the external edges by the $\mathrm{Y}, \mathrm{Z}$ axes as the mean coordinate of the atoms in this plane. Then we make an indentation of 0.25 angstrem in order to take into account that the atom is not a material point, but it has a certain volume. This indentation gives finally the substance (copper) density obtained experimentally. Thus we find the area of the extreme edges perpendicular to the $\mathrm{X}$ axis.
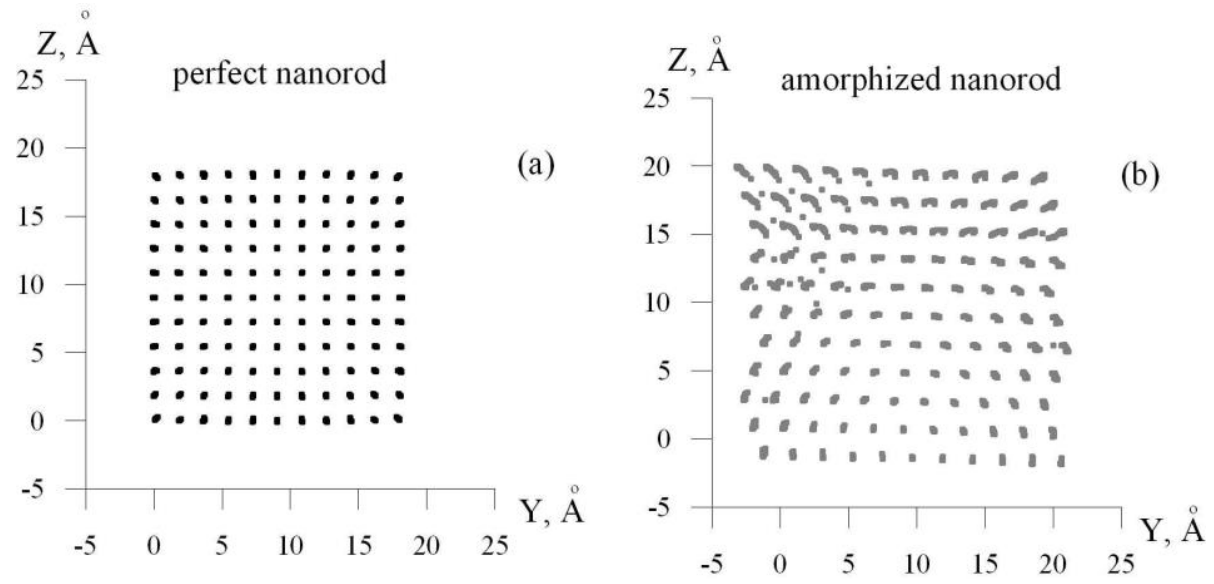

Fig. 2. View of the perfect (a) and amorphized (b) nanosized rod in the YZ plane. 
Let us compare both systems before the cyclic loading application. The common neighbor analysis gives the following results: the cooled perfect system (see Fig. 3) contains $65.6 \%$ atoms forming the face-centered cubic structure (FCC) and $34.4 \%$ surface atoms.

In the cooled amorphized nanorod (Fig. 4), there are only $35.6 \%$ atoms forming the face-centered cubic structure, and $35.9 \%$ surface ones and those which do not form the known crystal structure; there are $28.6 \%$ atoms forming the FCCP-lattice (face-centered close-packed lattice).
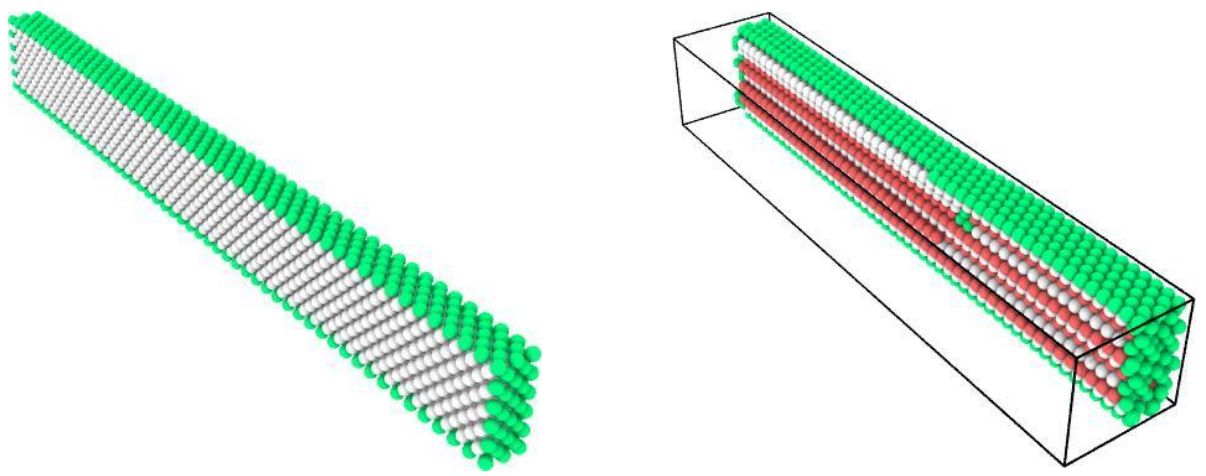

Fig. 3. (a) View of the perfect nanorod cut by a plane perpendicular to the $Y$ axis. Green shows surface atoms, white - the atoms in the volume. (b) View of the amorphized nanorod cut by a plane perpendicular to the $\mathrm{Y}$ axis. Green shows surface atoms, white shows the atoms in the volume forming the face-centered cubic structure; red shows the atoms forming the FCCP structure.
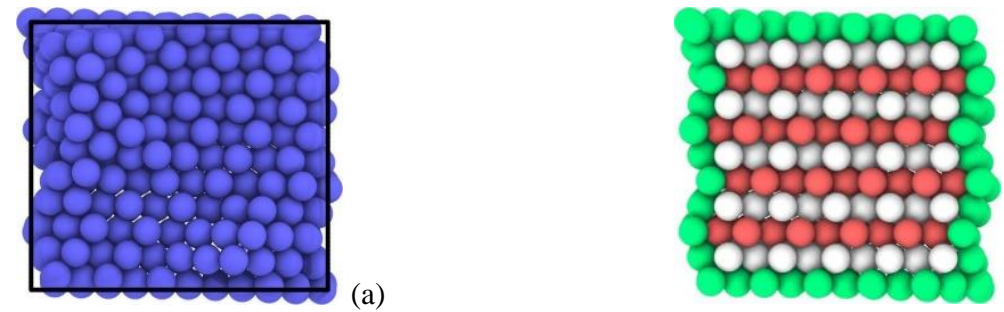

(b)

Fig. 4. (a) Outlook of the amorphized nanorod from the movable clamp side in the YZ plane. Blue indicates any atoms. (b) The cut of the amorphized nanorod by the plane perpendicular to the $\mathrm{X}$ axis. Green shows the surface atoms, white shows the atoms forming the face-centered cubic structure, red shows the atoms forming the FCCP structure.

Figure 5 demonstrates the internal structure of the amorphized nanorod resulting from the cut by the plane perpendicular to the $\mathrm{Y}$ axis. The common neighbor analysis of the crystal structure shows that the amorphized nanorod initially contains almost one third of the atoms forming the FCCP lattice. The general view of the amorphized nanorod differs significantly from the perfect nanorod, which shape approaches to the rectangular parallelepiped.

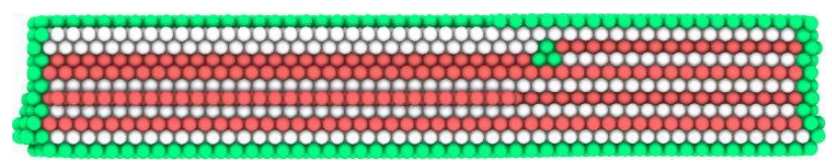

Fig. 5. Cut of the amorphized nanorod by the plane perpendicular to the $Y$ axis. Green shows the surface atoms, white shows the atoms forming the face-centered cubic structure, red shows the atoms forming the FCCP structure. 


\section{Comparison of energy absorption under the cyclic loading by the perfect and amorphized nanorods}

\section{1 $\Delta \mathrm{x}_{0}=0.5 \AA, v=0.085 \mathrm{THz}$}

With this set of data, the fracture of the perfect nanorod does not begin up to $500 \mathrm{ps}$. Let us construct the dependencies of the total system energy amplification and its components for the perfect and amorphized nanorods (Fig. 6).

As is seen from the Fig. 6, at this set of parameters of the cyclic loading, the amorphized nanorod absorbs the energy weaker.

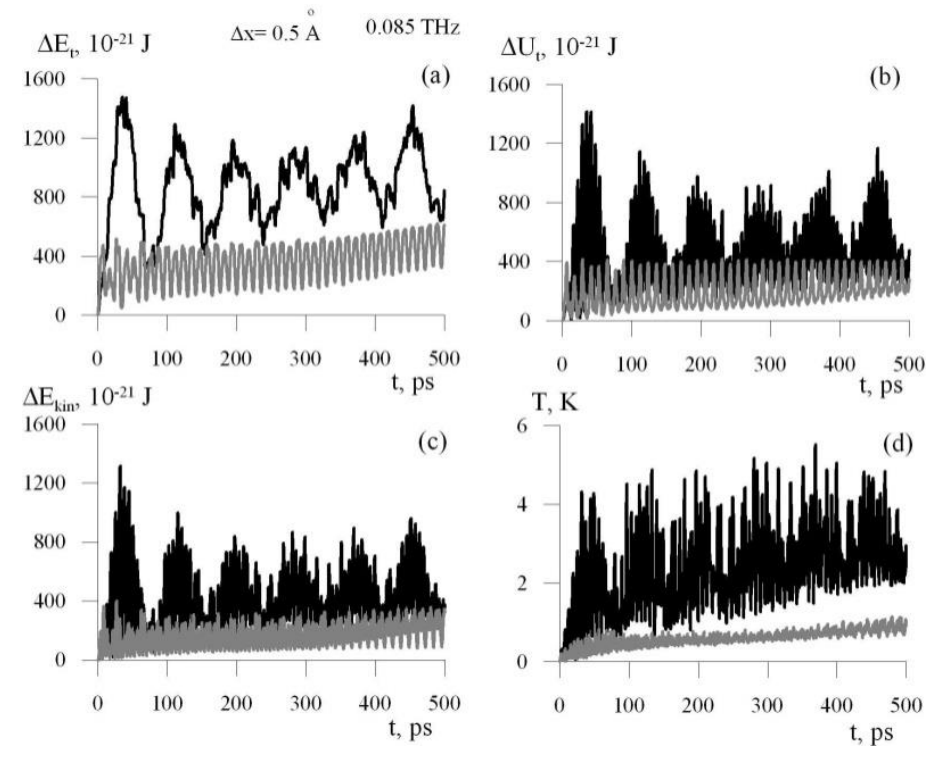

Fig. 6. Time dependence energy characteristics of the system under the cyclic loading: $\Delta \mathrm{x}_{0}=0.5 \AA$, $v=0.085 \mathrm{THz}$. (a) - amplification of the total energy of the system; (b) - amplification of the potential energy of atomic interaction; (c) - amplification of the thermal energy; (d) - the temperature found via the energy of thermal chaotic motion of atoms. Black line is the perfect crystal; grey line is the amorphized crystal.

\subsubsection{Maximal dispersion of atomic planes}

Figure 7 presents the time dependence of the maximal dispersion and variations of the maximal dispersion in respect to the same value in the undisturbed system.

Comparison of the amorphization effect by the maximal dispersion of atomic planes is not correct because at the very initial time instant before the external disturbance start, the dispersion of atomic planes in the amorphized crystale already exceeds essentially this parameter in the perfect nanorod. It is better to use for comparison the atomic planes dispersion deviation from its initial value (Fig. 7 (b)). 

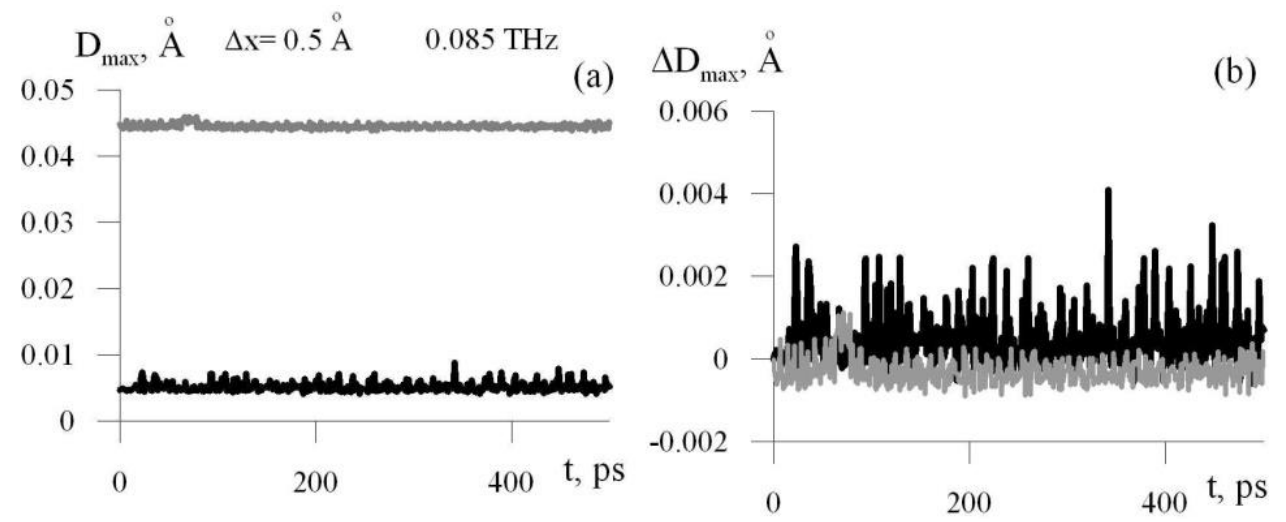

Fig. 7. Time dependence the maximal dispersion (а) и изменения the maximal dispersion (b) in respect to the same value in the undisturbed system. Black line - perfect crystal; grey line amorphized crystal. $\Delta \mathrm{x}_{0}=0.5 \AA, v=0.085 \mathrm{THz}$.

\subsubsection{Crystal structure analysis}

At the frequency $0.085 \mathrm{THz}$ and amplitude $0.5 \AA$, within 5 mio time pitches (500 ps), even a visible defect of the system is not observed in the perfect crystale. The common neighbor analysis demonstrates that there are some insignificant variations of the content structure, but they lie within the limits of a tenth of a percent (units of atoms). The situation is similar in the amorphizedom nanorod. It means that at these parameters of the cyclic loading, no fracture occurs in the systems.

At the same time, the temperature rises in the perfect nanorod, though weakly, which means the probable fracture in the case of the giga-cyclic long-term loading. In the amorphized nanorod, the temperature rises too, but even slower than in the perfect system.

\section{$4.2 \Delta \mathrm{x}_{0}=0.5 \AA, v=1 \mathrm{THz}$}

It is seen from the data in Fig. 8 that as the frequency rises, the character of absorption of every energy component changes. The oscillatory character with periodical pulsations (see Fig. 6) has transformed into the monotony growth of the absorbed energy.

With this parameter combination, the fracture of perfect nanorod, understood as the separation of the whole sample to fragments, does not occur up to $70 \mathrm{ps}$, which is shown below via the nanorod outlook visualization. Whereas the irreversible damage of the crystal structure appears by the $2^{\text {nd }}$ ps already. Below there are the dependencies of the amplification of the system total energy and its components for the nanorod with the initially perfect crystal structure (black line) and for amorphized nanorod (grey line) up to $12 \mathrm{ps}$, for better understanding of the difference in every characteristic behavior.

As the frequency rises, the energy absorption by the amorphized nanorod exceeds the energy absorption of the perfect structure (see the similar Fig. 6 for the frequencies $0.085 \mathrm{THz})$. 


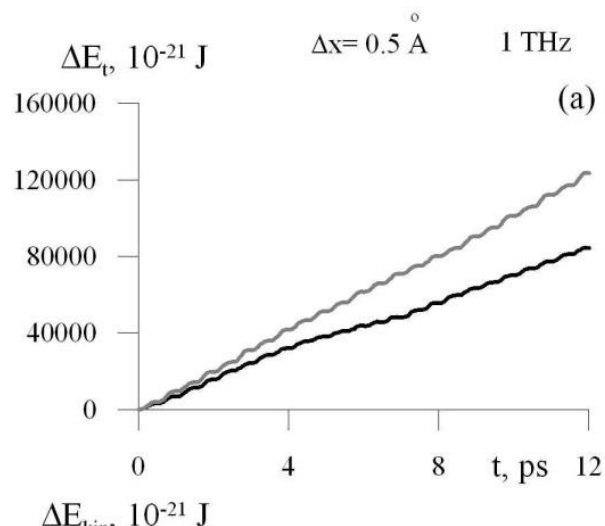

$$
\Delta \mathrm{U}_{\mathrm{t}}, 10^{-21} \mathrm{~J}
$$
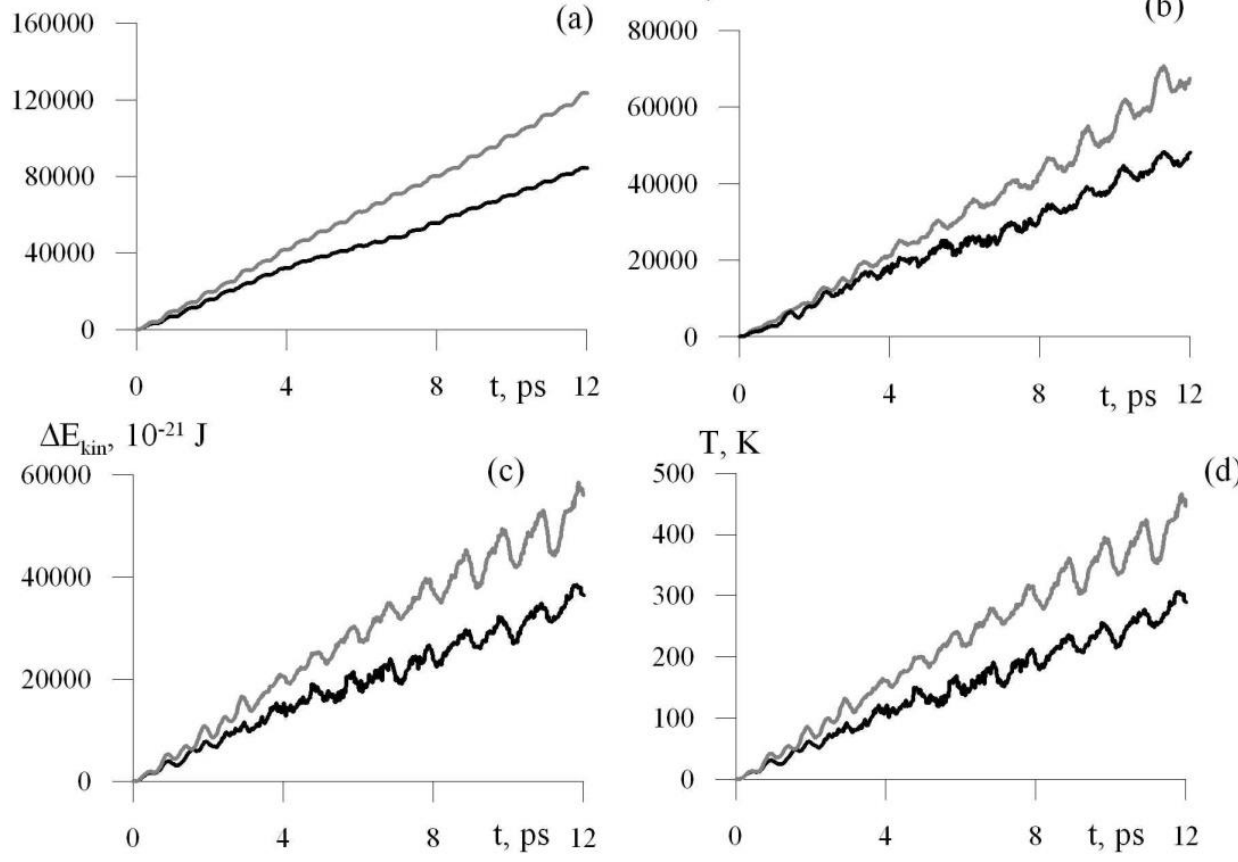

Fig. 8. Time dependence for the energy characteristics of the system under the cyclic loading: $\Delta \mathrm{x}_{0}=$ $0.5 \AA, v=1$ THz. (a) - amplification of the total energy of the system; (b) - amplification of the potential energy of atom interaction; (c) - amplification of the thermal energy; (d) - the temperature found via the energy of the thermal chaotic motion of atoms. Black line - perfect nanorod; grey line amorphized nanorod.

It is evident (Fig. 9) that in the amorphized nanorod, the temperature by the $70^{\text {th }} \mathrm{ps}$ after the start of the cyclic loading exceeds the copper melting point $(1356 \mathrm{~K})$ and reaches $1375 \mathrm{~K}$. It means that, with the combination of the above mentioned parameters of the cyclic loading: $\Delta \mathrm{x}_{0}=0.5 \AA, v=1 \mathrm{THz}$, the fracture occurs not because of the mechanical separation of the samples to fragments, but due to the system disturbance energy absorption, which causes the temperature growth (the energy of the chaotic motion of atoms). Note that this type of fracture is observed both for the initially perfect and for amorphized systems. 


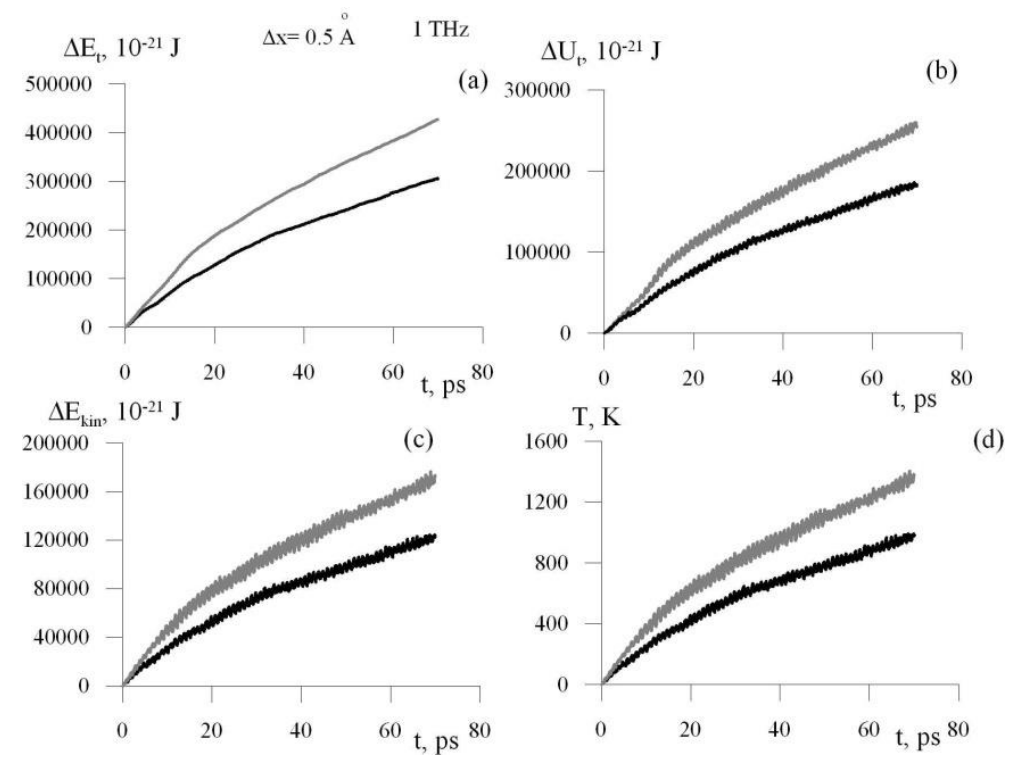

Fig. 9. Time dependence of the energy characteristics of the system: (a) - amplification of the total energy of the system; (b) - amplification of the potential energy взаимодействия atoms; (c) amplification of the thermal energy; (d) - the temperature found via the energy of the thermal chaotic motion of atoms. Black line - perfect nanorod; grey line - amorphized nanorod. $\Delta \mathrm{x}_{0}=0.5 \AA, v=1$ THz.

\subsubsection{Analysis of defects and fracture in the systems}

Figure 10 presents the outlook of the perfect and amorphized rods at the same time instant of $2 \mathrm{ps}$, when the essential damage of the crystal structure is visible in the area of the moving clamp (i.e. from the side of the cyclic loading application).

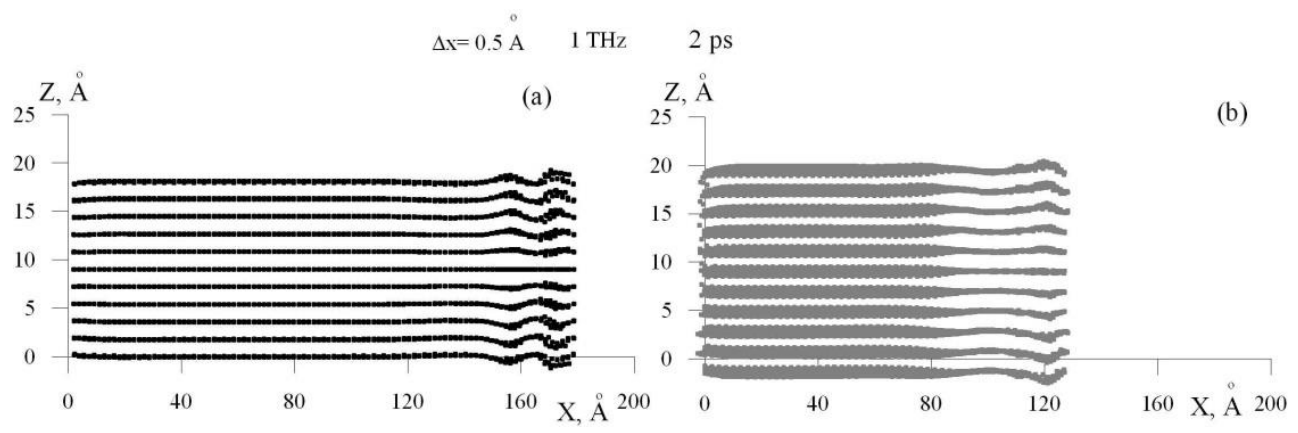

Fig. 10. Outlook of the initially perfect (a) and amorphized (b) nanorod at the time instant 2 ps. $\Delta \mathrm{x}_{0}=$ $0.5 \AA, v=1 \mathrm{THz}$.

Below there are both studied systems are presented at different cyclic action time instants, which shows successfully the development of the crystal structure damage which propagates from right, from the movable clamp side: 10, 20, 50, 70 ps for the cyclic loading parameters: $\Delta \mathrm{x}_{0}=0.5 \AA, \mathrm{v}=1 \mathrm{THz}$ (Figs. 11-14). 

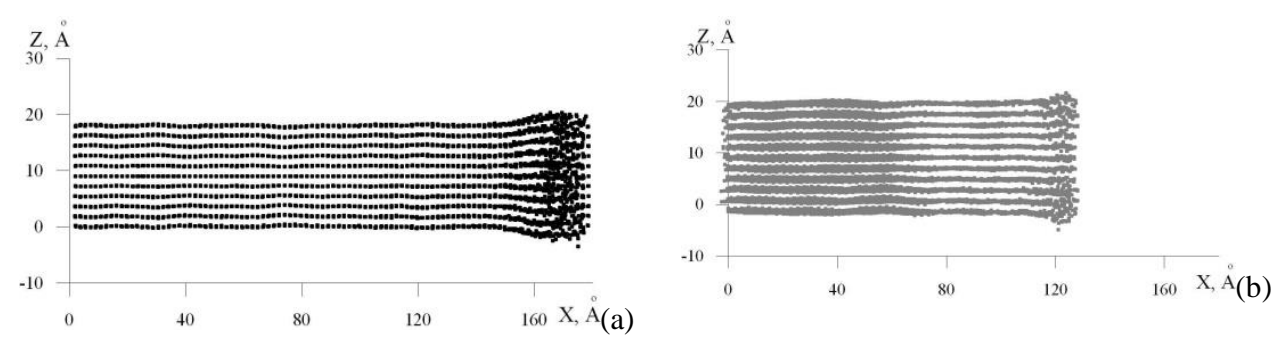

Fig. 11. View of the perfect (a) and amorphized (b) nanosized rod in XZ plane $10 \mathrm{ps}$ after the beginning of external cyclic action.
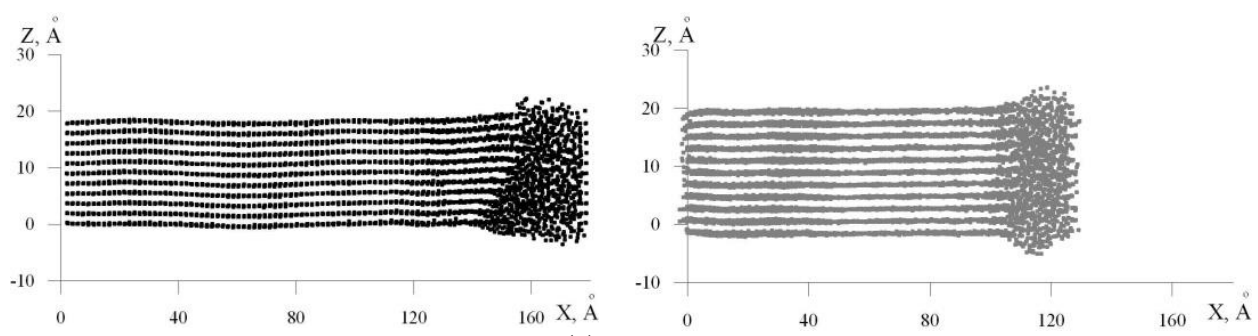

(a)

Fig. 12. Outlook of the initially perfect nanorod (a) at the time instant $20 \mathrm{ps}$ and amorphized nanorod (b).
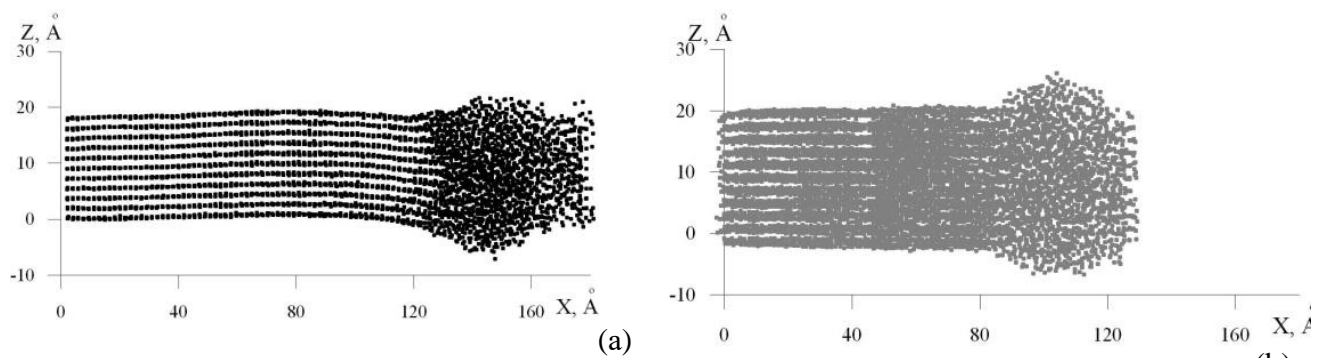

(a)

(b)

Fig. 13. Outlook of the initially perfect nanorod (a) at the time instant $50 \mathrm{ps}$ and amorphized nanorod (b).
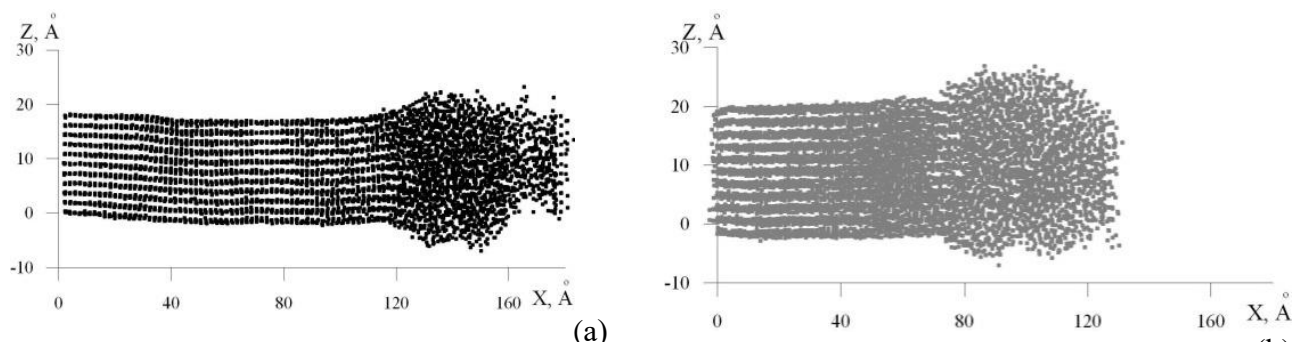

(a)

Fig. 14. Outlook of the initially perfect nanorod (a) at the time instant $70 \mathrm{ps}$ and amorphized nanorod (b).

\subsubsection{Maximal dispersion of atomic planes}

Figure 15 presents the time dependence of the maximal dispersion of atomic planes and varying maximal dispersion in respect to the same value in the undisturbed system. The variations are similar. 


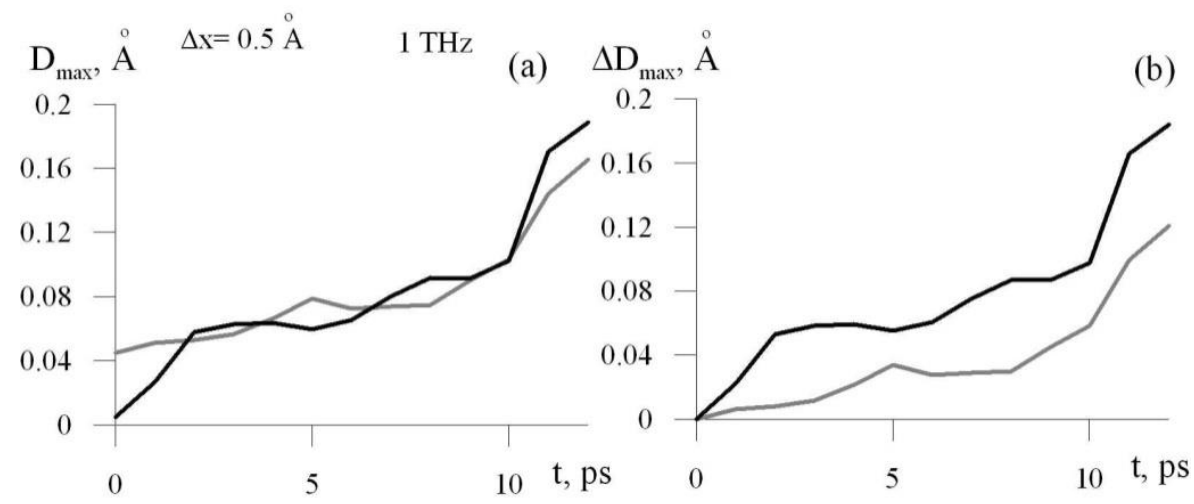

Fig. 15. Maximal dispersion of atomic planes versus time (a) and variations of the maximal dispersion in respect to the same value in the undisturbed system (b). Black line - perfect crystal, grey line - amorphized system. $\Delta \mathrm{x}_{0}=0.5 \AA, \mathrm{v}=1 \mathrm{THz}$.

\subsubsection{Analysis of the crystal structure}

Now let us compare the variations of both systems structures by the common neighbor analysis, with the aid of the visualization program OVITO (htto://ovito.org/).

Let us consider the nanorod with the initially perfect crystal structure at the time instant 2 ps after the cyclic loading start (Fig. 16).

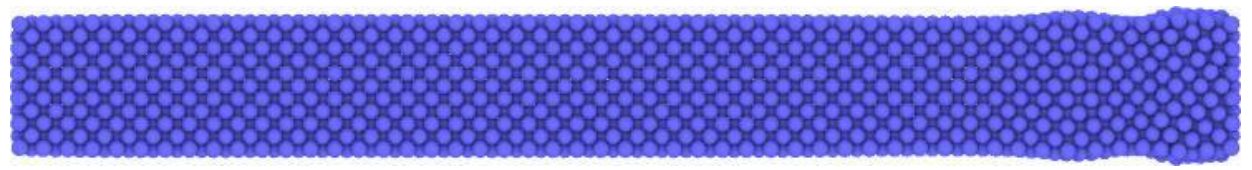

Fig. 16. Outlook of the initially perfect nanorod at the time instant $2 \mathrm{ps}$ after the cyclic loading start. $\Delta \mathrm{x}_{0}=0.5 \AA, v=1 \mathrm{THz}$.

Figure 17 shows the cut of this system by the plane perpendicular to the $\mathrm{Y}$ axis. The atoms constructing different crystal structure have different colors.

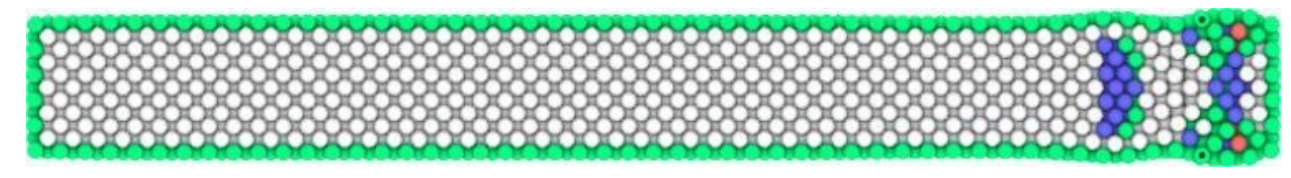

Fig. 17. Cut of the initially perfect nanorod by the plane perpendicular to the $Y$ axis, at the time instant 2 ps after the cyclic loading start. $\Delta \mathrm{x}_{0}=0.5 \AA, v=1 \mathrm{THz}$. Green shows the surface atoms and other structureless ones; white shows the atoms forming the FCC lattice, blue shows the atoms forming the BCC lattice, and red shows the atoms forming the FCCP lattice.

In the Fig. 18 there is the cut of the nanorod by the plane perpendicular to the $\mathrm{X}$ axis at the time instant 2 ps after the cyclic loading start. The cut is made within about $5 \AA$ from the rod movable clamp. It is evident that the crystal structure transformation is symmetrical under the cyclic loading action. Figure 18 (b) shows the place where the cut is made. 

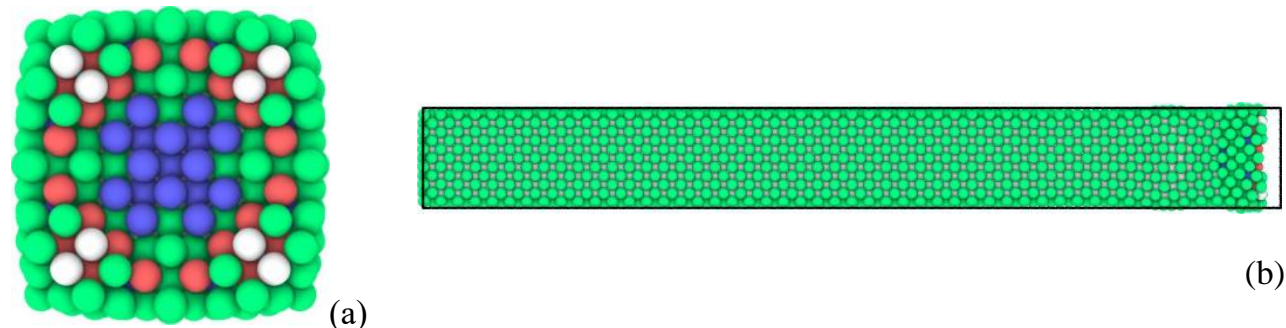

(a)

(b)

Fig. 18. (a) Cut of the initially perfect nanorod by the plane perpendicular to the $\mathrm{X}$ axis within $5 \AA$ from the right edge at the time instant 2 ps. Green shows the surface atoms and other structureless ones; white shows the atoms forming the FCC lattice; blue shows the atoms forming the BCC lattice; red shows the atoms forming the FCCP lattice. (b) Outlook of the nanorod in plane XZ, black line the whole system size, the cut area is to the right within $5 \AA$ from the right edge.

Let us compare what happens in the amorphized nanorod at the same time instant 2 ps after the cyclic loading start (Figs. 19 and 20).

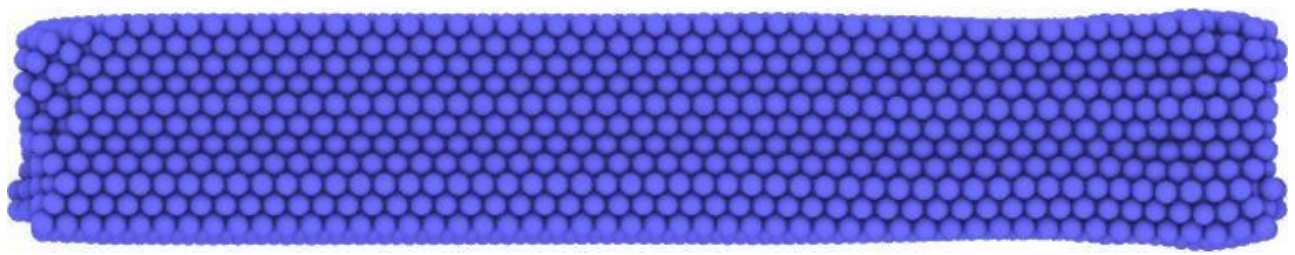

Fig. 19. Outlook of the amorphized nanorod at the time instant $2 \mathrm{ps}$ after the cyclic loading start. $\Delta \mathrm{x} 0$ $=0.5 \AA, v=1 \mathrm{THz}$.

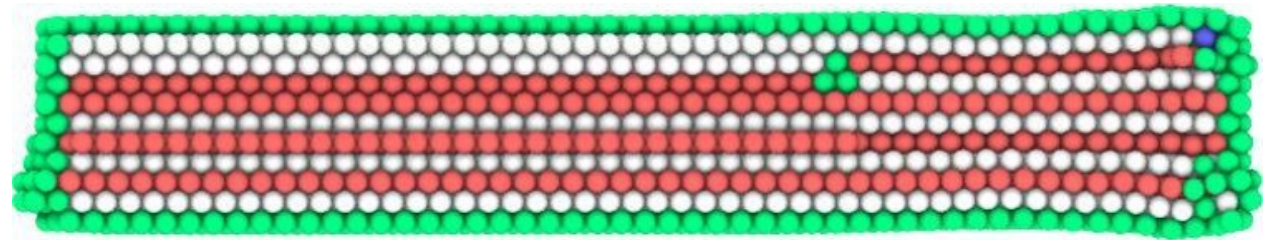

Fig. 20. Cut of the amorphized nanorod by the plane perpendicular to the $\mathrm{Y}$ axis, at the time instant 2 ps after the cyclic loading start. $\Delta \mathrm{x}_{0}=0.5 \AA, v=1 \mathrm{THz}$. Green shows the surface atoms and other structureless ones; white shows the atoms forming the FCC lattice; blue shows the atoms forming the BCC lattice; red shows the atoms forming the FCCP lattice.

It is seen that in the amorphized system under the cyclic loading, there are fewer variations in the crystal structure than in the initial one: compare Fig. 5 and Fig. 20. Then there is the cut of the nanorod by the plane perpendicular to the $\mathrm{X}$ axis at the time instant 2 ps (Fig. 21). It is evident that the crystal structure transformation develops nonsymmetrically under the cyclic loading action.

Let us compare which structural changes have appeared in the studied systems by the $10^{\text {th }} \mathrm{ps}$. Figure 22 shows that the total structure amorphization occurs in the initially perfect system within $5 \AA$ from the movable clamp. 

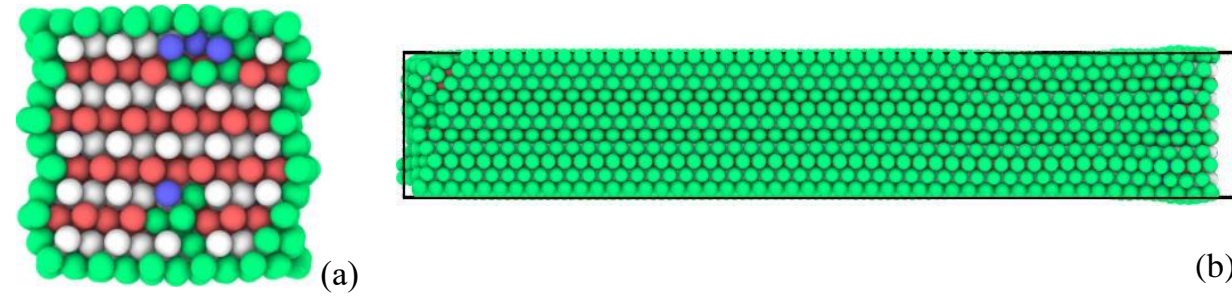

(a)

(b)

Fig. 21. (a) Cut of the nanorod by the plane perpendicular to the axis $X$ within $5 \AA$ from the right edge at the time instant 2 ps. Green shows the surface atoms and other structureless ones; white shows the atoms forming the FCC lattice; blue shows the atoms forming the BCC lattice; red shows the atoms forming the FCCP lattice. (b) Outlook of the nanorod in plane XZ, black line - the whole system size, the cut area is to the right within $5 \AA$ from the right edge.

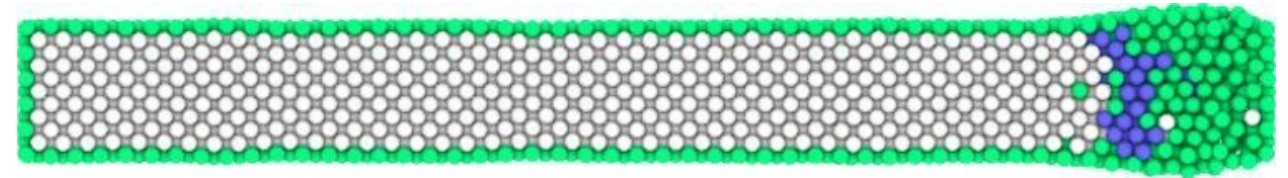

Fig. 22. Cut of the initially perfect nanorod by the plane perpendicular to the $Y$ axis, at the time instant $10 \mathrm{ps}$ after the cyclic loading start. $\Delta \mathrm{x}_{0}=0.5 \AA, v=1 \mathrm{THz}$. Green shows the surface atoms and other structureless ones; white shows the atoms forming the FCC lattice; blue shows the atoms forming the BCC lattice.

In general, the damage under the cyclic loading develops in the way similar to the process in the initially perfect and amorphized nanorods.

By the time instant of 10 ps (Fig. 23), the disturbance wave, which propagation appears itself qualitatively via the varying crystal structure is respect to the initial one, shifted even further from the moving clamp.

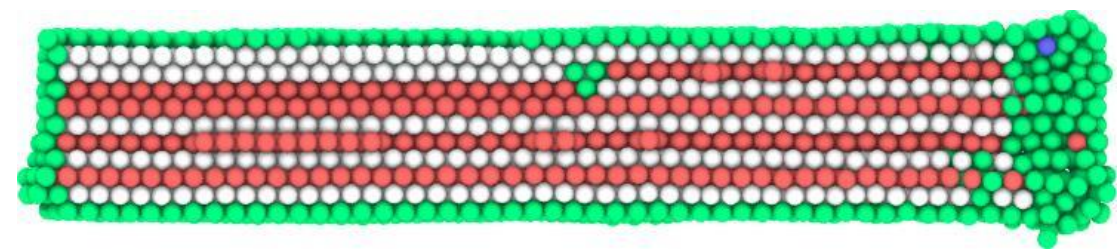

Fig. 23. Cut of the amorphized nanorod by the plane perpendicular to the $\mathrm{Y}$ axis, at the time instant $10 \mathrm{ps}$ after the cyclic loading start. $\Delta \mathrm{x}_{0}=0.5 \AA, v=1 \mathrm{THz}$. Green shows the surface atoms and other structureless ones; white shows the atoms forming the FCC lattice; blue shows the atoms forming the BCC lattice; red shows the atoms forming the FCCP lattice.

\section{$4.3 \Delta \mathrm{x}_{0}=0.5 \AA, v=10 \mathrm{THz}$}

Since at this set of parameters by the $2^{\text {nd }}$ ps the fracture of the initially perfect system occurs [7], we present the data reflecting the system disturbance energy absorption only before 2 ps (Fig. 24).

\subsubsection{Analysis of defects and fracture in the systems}

Outlook of the studied systems at the time instant 2 ps after the cyclic loading start shows the presence of the fracture is both systems (Fig. 25). In the amorphized system, the fracture is visually more developed by the $2^{\text {nd }} \mathrm{ps}$. 


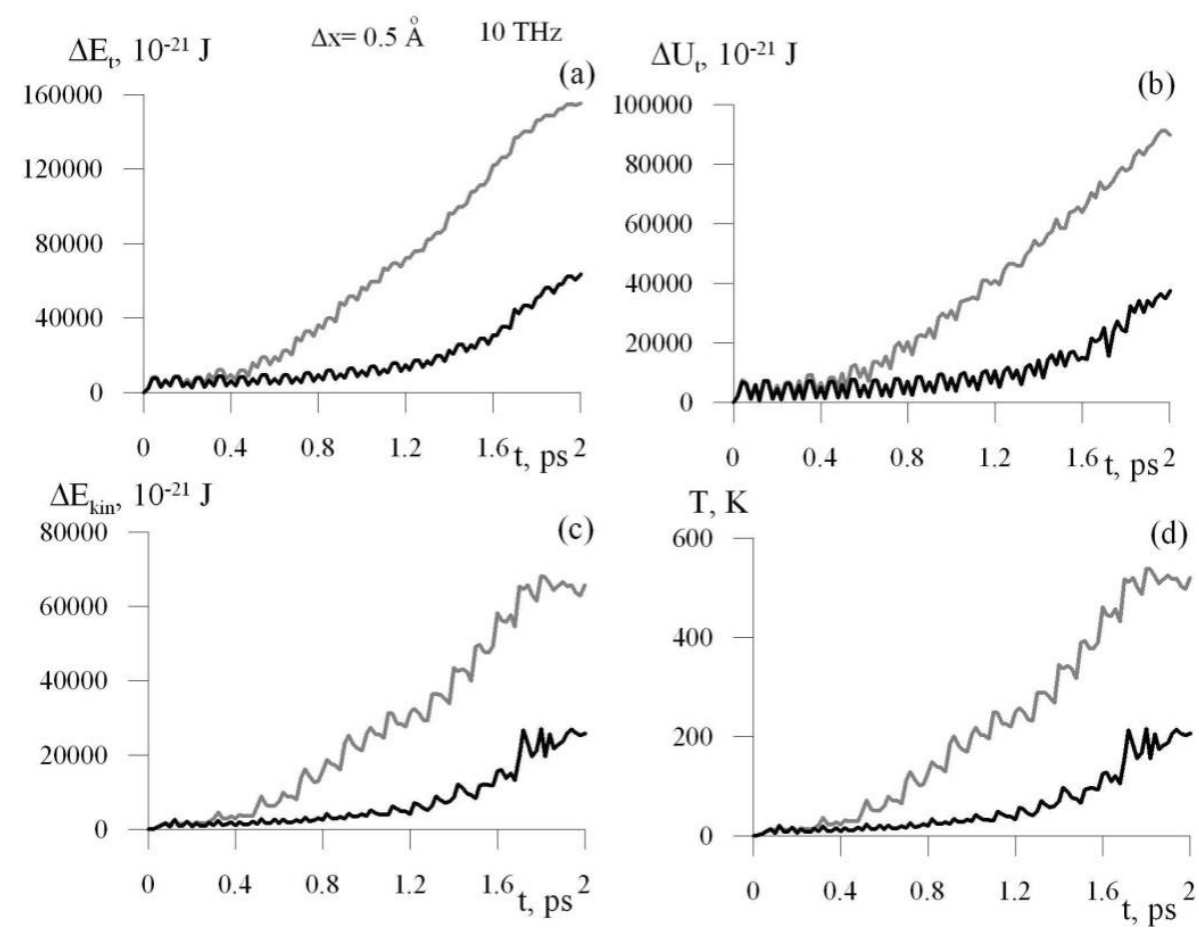

Fig. 24. Amplification of the total energy (a) and its components: potential (b) and kinetic (c), system temperature (d) versus time. Black line - perfect crystal, grey line - amorphized system. $\Delta \mathrm{x}_{0}=0.5 \AA$, $v=10 \mathrm{THz}$.
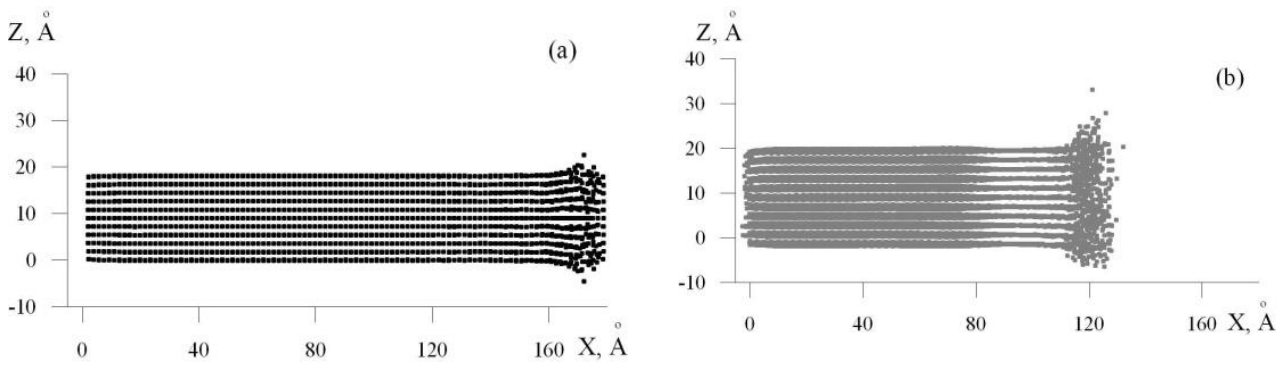

Fig. 25. Outlook of the initially perfect nanorod (a) and amorphized nanorod (b) at the time instant 2 ps after the cyclic loading start $\left(\Delta \mathrm{x}_{0}=0.5 \AA, v=10 \mathrm{THz}\right)$.

\subsubsection{Maximal dispersion of atomic planes}

Below there is the time dependence of the maximal dispersion of atomic planes and maximal dispersion amplification in respect to the same value in the undisturbed system for perfect and amorphized systems (Fig. 26).

It is seen from Fig. 26 that very similar behavior of atomic planes dispersion and dispersion amplification is observed both in the perfect and amorphized systems. 

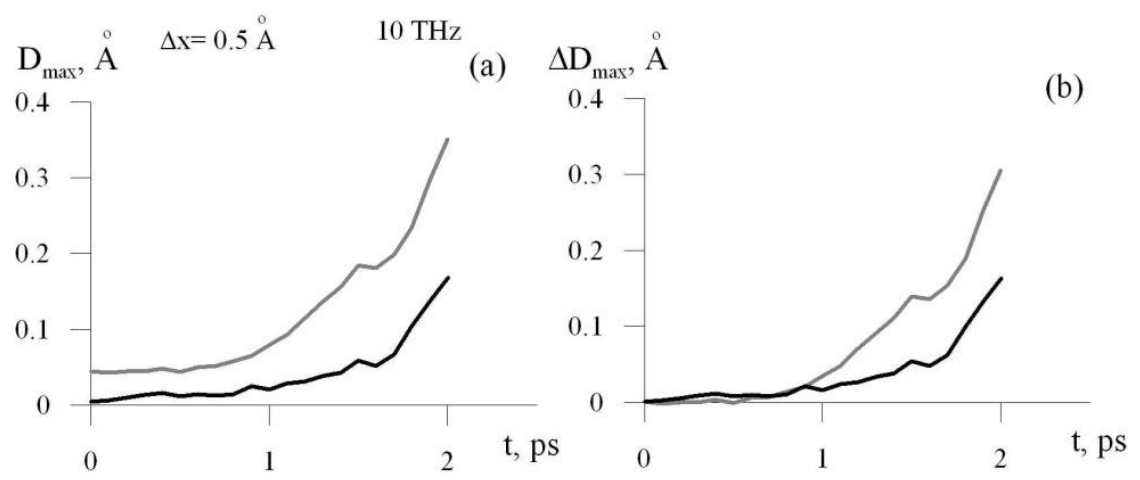

Fig. 26. Maximal dispersion versus time (a) and variations of the maximal dispersion in respect to the same value in the initial system before the external action start (b). Black line - perfect crystal, grey line - amorphized system. $\Delta \mathrm{x}_{0}=0.5 \AA, v=10 \mathrm{THz}$.

\section{Conclusions}

- Both the dramatic growth of the maximal dispersion of atomic planes, and the dramatic growth of amplification of the maximal dispersion of atomic planes can be treated as an index of the irreversible transformation of the crystal structure in the physical system differing from the perfect nanosized crystal.

- General behavior of the studied systems depends more on the cyclic loading parameters than on the object crystal structure and size.

- At the frequency $0.085 \mathrm{THz}$ (which is proper for this perfect basic nanorod), the energy absorption of the system with the initially perfect crystal structure exceeds the energy absorption of the amorphized system. But beginning from the frequency $1 \mathrm{THz}$, the amorphized system absorbs the cyclic loading energy more intensively than the initially perfect system.

- Under the cyclic loading modeled by the periodical shift of the movable clamp, the frequency imposes the critical effect on the results.

This work is financially supported by the Russian Foundation for Basic Research, Russia, grant No. 17-01-00068. "The research of temperature influence on the fatigue fracture of metal nanostructures» and it was partly carried out within the framework of the Program of Fundamental Scientific Research of the state academies of sciences in 2013 - 2020 (project No. AAAA-A17117030610134-9 «The mechanics of deformation and fracture of materials, media, under mechanical loads, exposure to physical fields and chemically active media»).

\section{References}

1. I.F. Golovnev, E.I. Golovneva, A.V. Utkin, Procedia Structural Integrity 13, 16321637 (2018)

2. I.F. Golovnev, E.I. Golovneva, L.A. Merzhievsky, V.M. Fomin, Phys. Mesomech. 16, 4, 294 - 302 (2013)

3. I.F. Golovnev, E.I. Golovneva, L.A. Merzhievsky, V.M. Fomin., and V.E. Panin, Phys. Mesomech., 18, 3, 179 - 186 (2015)

4. A.F. Voter, Los Alamos Unclassified Technical Report (\# LA-UR 93-3901, 1993)

5. I.F. Golovnev, E.I. Golovneva, V.M. Fomin, Phys. Mesomech. 6, 5 - 6, 41 - 45 (2003) 
6. M.P. Allen, D.J. Tildesley, Computer Simulation of Liquids (New York: Clarendon Press, 1987)

7. I. Golovnev, E. Golovneva, A. Utkin, Engineering Failure Analysis 105, 672 - 687 (2019)

8. A. Stukowski, Modell. Simul. Mater. Sci. Eng. 18, 015012 (2010)

9. A. Stukowski, Modell. Simul. Mater. Sci. Eng. 20, 045021 (2012) 